[10] W. R. Johnson, K. T. Cheng, K. N. Huang, and M. Le Dourneuf, "Analysis of Beutler-Fano autoionizing resonances in the rare-gas atoms using the relativistic multichannel quantum-defect theory," Phys. Rev. A, vol. 22, pp. 989-997, 1980.

[11] D. J. Jackson and J. J. Wynne, "Interference between different optical harmonics," Phys. Rev. Lett., vol, 49, pp. 543-546, 1982.

[12] J. H. Glownia and R. K. Sander, "Experimental evidence for the competition between resonantly enhanced multiphoton ionization and third-harmonic generation in xenon," Phys. Rev. Lett., vol. 49, pp. 21-24, 1982.

[13] J. C. Miller and R. N. Compton, "Third-harmonic generation and multiphoton ionization in rare gases," Phys. Rev. A, vol. 25, pp. 2056-2063, 1982.

[14] E. Yablonovitch, C. Flytzanis, and N. Bloembergen, "Anisotropic interference of three-wave and double two-wave frequency mixing in GaAs," Phys. Rev. Lett., vol. 29, pp. 865-868, 1972.

[15] I. V. Tomov and M. C. Richardson, "Fifth-harmonic generation in isotropic media," IEEE J. Quantum Electron., vol. QE-12, pp. 521-531, Sept. 1976.

[16] D. C. Hanna, M. A. Yuratich, and D. Cotter, Nonlinear Optics of Free Atoms and Molecules. Berlin, Germany: Springer-Verlag, 1979, p. 119.

[17] F. Vallée, S. C. Wallace, and J. Lukasik, "Tunable coherent vacuum ultraviolet generation in carbon monoxide in the 1150 \& range," Opt. Commun., vol. 42, pp. 148-150, 1982.

[18] F. Vallée and J. Lukasik, "Vacuum ultraviolet generation in phasematched carbon monoxide," Opt. Commun., vol. 43, pp. 287$291,1982$.

\title{
Field and Hot Carrier Enhanced Leakage in InGaAsP/InP Heterojunctions
}

\author{
L. C. CHIU, K. L. YU, S. MARGALIT, T. R. CHEN, U. KOREN, A. HASSON, AND AMNON YARIV
}

\begin{abstract}
A model calculation for the field and hot carrier enhanced electron leakage in InGaAsP/InP LED's and lasers is presented. The significant influence of the doping level in the P-InP confining layer on leakage current is confirmed.
\end{abstract}

$\mathrm{T}$ HE temperature sensitivity and power saturation of InGaAsP lasers and light emitting diodes (LED's) have triggered an extensive search for the responsible mechanisms. Prime candidates are nonradiative Auger recombination and carrier leakage over the heterobarrier. These are believed to be important factors affecting the performance of the family of quaternary optoelectronic devices emitting in the spectral range of 1.1-1.6 $\mu \mathrm{m}$. A number of new structures have been de. signed to isolate and thus directly demonstrate the role played by carrier leakage in LED's [1], [2] and lasers [3].

An interesting experimental observation of Chen et al. [3] is the continued increase of leakage current with total current above lasing threshold. Leakage models based on diffusion of electrons [4]- [6] alone cannot explain the observed phenomenon, as clamping of carrier density leads to a constant leakage current above lasing threshold. However, the observed behavior can be explained by invoking the electric field dominated drift component of electron current in the cladding layer. Lee and Dentai had included the field term in their calculation of the carrier confinement factor in $\mathrm{GaAs} / \mathrm{GaAlAs}$ LED's [7], but Anthony and Schumaker [8], [9] were the first to recognize the significance of this field. However, their theory predicted a linear dependence of leakage current on in-

Manuscript teceived December 16, 1982; revised April 5, 1983. This work was supported by the U.S. Office of Naval Research and the National Science Foundation.

The authors are with the California Institute of Technology, Pasadena, CA 91125 . jection current above threshold, contrary to the experimental result of [3]. The observed result can be explained by a theory analogous to the minority carrier injection in a Schottky diode [10], when the band discontinuity at the heterojunction is taken into account. We will show that in the high injection regime where lasers normally operate, the electron leakage current $J_{e}$ will be proportional to $J_{p}{ }^{\alpha}$, the injected hole current where $\alpha$ is a positive number between 2 and 3 . This would result in an "unclamped" leakage current which increases superlinearly with total injection current beyond lasing threshold. In this work, we present an electron leakage over the heterobarrier which incorporates the influence of both the electric field and carrier heating effect [11], [12].

Under high injection conditions in double heterostructure InGaAsP LED's and lasers, if the doping level in the P-InP cladding layer is low, it can easily be shown that there exists a substantial electric field across the InP layer. In such a case, the hole current can be written as

$$
\vec{J}_{p}=e \mu_{p} N_{A} \vec{E}
$$

where $e$ is the electric charge, $\mu_{P}$ the hole mobility, $N_{A}$ the doping level in the P-InP layer, and $\vec{E}$ the electric field strength. For simplicity, only the component transverse to the junction will be considered. The ratio of the drift and diffusion components of the electron leakage current across the p-InGaAsP$\mathrm{P}$-InP heterojunction is then given approximately by

$$
\frac{J_{\text {drift }}}{J_{\text {diff }}} \approx\left(\frac{\mu_{N}}{\mu_{P}}\right) \frac{L}{e D_{N} N_{A}} J_{p}
$$

where $L$ is the thickness of the cladding layer, and is assumed to be small compared to the diffusion length of electrons in the $\mathrm{P}$ cladding layer, $\mu_{N}$ the electron mobility, and $D_{N}$ the 
electron diffusion constant. Taking $\mu_{N}=3000 \mathrm{~cm}^{2} / \mathrm{V} \cdot \mathrm{s}, \mu_{P}=$ $150 \mathrm{~cm}^{2} / \mathrm{V} \cdot \mathrm{s}$ [13], $L=1.5 \mu \mathrm{m}, D_{N}=77 \mathrm{~cm}^{2} / \mathrm{s}$ (at $300 \mathrm{~K}$ ), and $N_{A}=10^{17} \mathrm{~cm}^{-3}$, it is easily verified that the drift current is larger than the diffusion current when $J_{p}$ exceeds $\sim 420$ $\mathrm{A} / \mathrm{cm}^{2}$. Since normal lasers operate at much higher current densities, it is expected that the drift rather than diffusion current will dominate. Since the drift (leakage) current increases with the field $\vec{E}$, and $\vec{E}$ increases with $J_{p}$ [see (1)], it follows that the leakage current continues to grow with current past threshold.

Fig. 1 shows the band structure at the p-InGaAsP-P-InP heterointerface at thermal equilibrium. $k$, the fraction of the diffusion potential $\left(V_{D}\right)$ on the widegap side, is given by [14]

$$
\kappa=\frac{1}{1+\left(\epsilon_{2} N_{A 2} / \epsilon_{1} N_{A 1}\right)}
$$

where $\epsilon$ is the dielectric constant and the subscripts 1 and 2 denote the low and high gap materials, respectively. The hole current under a forward bias $V\left(=V_{1}+V_{2}, V_{2}=\kappa V, V_{1}=(1-\kappa) V\right)$ is then given by

$$
\begin{aligned}
J_{P} & =\tilde{J}_{p s}\left(e^{e V_{2} / k T}-e^{-\left(e V_{1} / k T\right)}\right) \\
& =\tilde{J}_{p s} e^{-\left(e V_{1} / k T\right)}\left(e^{e V / k T}-1\right) \\
& =J_{p s}\left(e^{e V / k T}-1\right)
\end{aligned}
$$

with

$$
\begin{aligned}
J_{p s}= & e N_{A 2}\left(\frac{k T}{2 m_{2 h} \pi}\right)^{1 / 2} \frac{1}{\left(1+\frac{\left(k T / 2 m_{2 h} \pi\right)^{1 / 2}}{V_{d 2}}\right)} \\
& \cdot e^{-\left(e V_{1} / k T\right)} e^{-\left(k e V_{D} / k T\right)}
\end{aligned}
$$

where

$$
\begin{aligned}
V_{d 2}^{-1}= & \frac{1}{\mu_{P 2} k T} e^{\left[(1-\kappa) e V_{D}\right] / k T} e^{-\left(\Delta E_{v} / k T\right)} \\
& \cdot \operatorname{erf}\left[\left(\frac{\kappa e V_{D}}{k T}\right)^{1 / 2}\right] \sqrt{\frac{\pi \epsilon_{2} k T}{2 N_{A 2}}}
\end{aligned}
$$

which are exact analogues of the thermionic-diffusion model of a Schottky barrier [15]. In the previous equations, $m_{2 h}$ is the hole effective mass and $\Delta E_{v}$ is the discontinuity in the valence band. The electron concentration at $x_{2}$ (see Fig. 1) is given approximately by

$$
\begin{aligned}
N_{2}\left(x_{2}\right)= & \left(\frac{m_{2 e}}{m_{1 e}}\right)^{3 / 2} n_{1} \exp \left[\sum_{i=1}^{4} A_{i}\left(\frac{n_{1}}{N_{c 1}}\right)^{i}\right] \\
& \cdot \exp \left(-\frac{\Delta E_{c}}{k T}\right) \exp \left(\frac{e V_{D}}{k T}\right) \exp \left[\left(\frac{e V}{k T}\right)-1\right]
\end{aligned}
$$

where $n_{1}$ is the electron density in material 1 (InGaAsP), $A_{i}$ 's are constants which account for the degeneracy [14], and $N_{c 1}$ and $N_{c 2}$ are the conduction band effective density of states.

In most cases, it can be verified that $v_{d} \gg\left(k T / 2 m_{2 n} \pi\right)^{1 / 2}$, and the conduction mechanism is thermionic emission dominated [16]. Also, though (4) indicates a rectifying characteristic, the large $J_{p s}$ value (which exceeds $1000 \mathrm{~A} / \mathrm{cm}^{2}$ in most
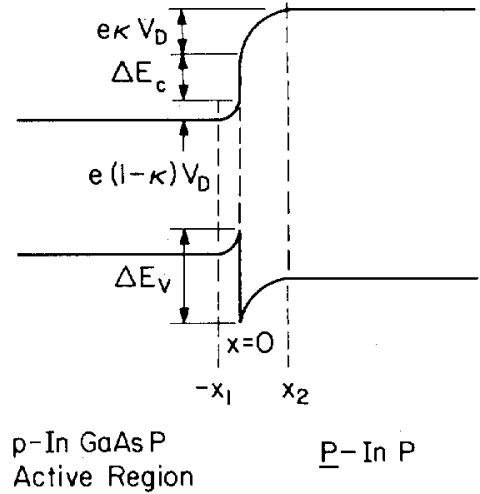

Fig. 1. Band structure at the p-InGaAsP-P-InP heterointerface at thermal equilibrium.

cases) renders the junction more "ohmic" than rectifying in nature.

Solving the carrier transport and continuity equations for electrons, the electron leakage current is given by [10]

$$
\begin{aligned}
J_{e}(x)= & \left(\frac{\mu_{N}}{\mu_{P}}\right) \frac{J_{p}}{N_{A 2}} N_{2}\left(x_{2}\right)-\frac{e D_{N}}{A} N_{2}\left(x_{2}\right) \\
& \cdot\left[\frac{1}{y_{1}} e^{L / y_{2}-x / y_{1}}+\frac{1}{y_{2}} e^{-\left(L / y_{1}\right)+x / y_{2}}\right]
\end{aligned}
$$

where

$$
\begin{aligned}
& A=e^{x_{2} / y_{2}-L / y_{1}}-e^{-\left(x_{2} / y_{1}\right)+L / y_{2}} \\
& y_{\frac{1}{2}}=\frac{ \pm L_{e d}^{2}+\sqrt{L_{e d}^{4}+4 L_{e}^{2}}}{2} \\
& L_{e d}^{2}=\left(\frac{\mu_{N}}{\mu_{P}}\right) \frac{J_{p} \tau_{N 2}}{e N_{A 2}}, \quad \text { and } \quad L_{e}^{2}=D_{N} \tau_{N 2}
\end{aligned}
$$

with $\tau_{N 2}$ the electron lifetime in the InP cladding layer. In arriving at the above solution, we have assumed the boundary condition of $N_{2}(L)=0$. However, when the leakage is drift dominated, the boundary condition $N_{2}(L)$ is not important, as is apparent from (8).

The total injected current density $J_{\text {tot }}$ is simply

$$
J_{\text {tot }}=J_{p}+J_{e}=J_{\text {rec }}+J_{\text {leak }} \text {. }
$$

Since $J_{e}$ is just the leakage current $J_{\text {leak }}$, it follows that the recombination current $J_{\text {rec }}=J_{p}$. Up to lasing threshold, $J_{p}$ can be expressed as

$$
\begin{aligned}
\frac{J_{\mathrm{rec}}}{e d}= & B n_{1}\left(n_{1}+N_{A 1}\right)+C_{\mathrm{chcc}} n_{1}^{2.09}\left(n_{1}+N_{A 1}\right) \\
& +C_{\mathrm{chsh}} n_{1}\left(n_{1}+N_{A 1}\right)^{2.14}
\end{aligned}
$$

where $d$ is the active layer thickness, $B$ the radiative recombination constant, $C_{\text {chce }}$ and $C_{\text {chsh }}$ are the Auger coefficients for the $\mathrm{CHCC}$ and CHSH processes [17]. $B$ is estimated from spontaneous lifetime data and $C_{\text {chcc }}$ and $C_{\text {chsh }}$ are calculated as described in [17] .

As can be seen from (4)-(7), the discontinuities in the conduction $\left(\Delta E_{c}\right)$ and valence band $\left(\Delta E_{v}\right)$ play an important role in determining the magnitude of the leakage current. Unfor- 


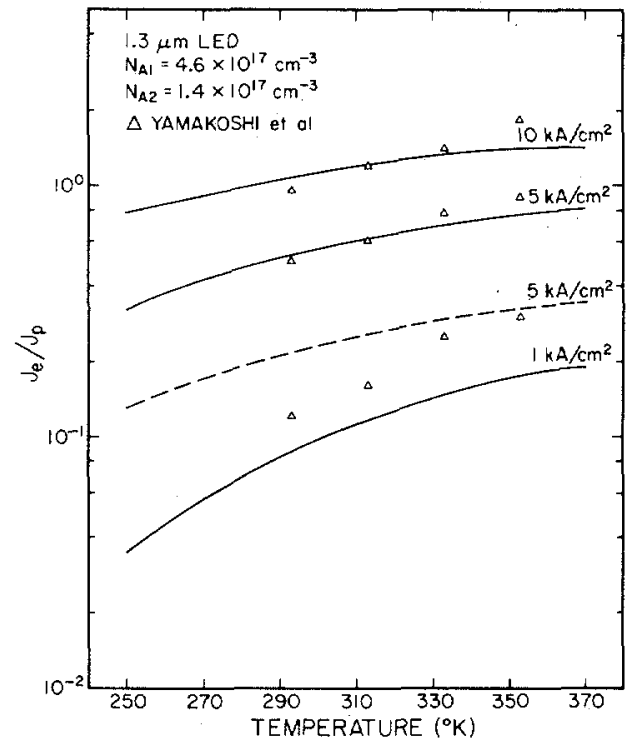

Fig. 2. Calculated $J_{e} / J_{p}$ under different injection conditions. Experimental data are from [2]. The dashed line denotes the result when hot carrier effect is neglected.

tunately, the discontinuity in the quaternary sys ${ }^{`} \mathrm{~m}$ is not accurately known. However, there are experimental [18], [19], and theoretical [20] indications that a substantial fracion of the discontinuity lies in the valance band. We have used $\Delta E_{c}=\frac{1}{3} \Delta E_{g}$ as measured in [18] and [19]. If the interface is graded, then the potential "spike" which is resistive to hole injection would decrease, resulting in less leakage. In this work, an abrupt interface is assumed. However, the ratio $\Delta E_{c} / \Delta E_{v}$ as well as the doping level $N_{A 2}$ are varied to provide a quantitative trend and estimate of the leakage current and its effects.

It was found that for $1.3 \mu \mathrm{m}$ LED's, if effects of carrier heating and/or Auger enhanced leakage [21] were excluded, the predicted leakage using reasonable parameters would be much smaller than the observed values. Much better agreement could be obtained by incorporating carrier heating effect or employing a boundary condition $n_{2}\left(x_{2}\right) \sim \exp \left[-\frac{3}{4}\left(\hbar \omega_{0} / E\right)\right.$. $\left.\left(d^{2} / L_{P}^{2}\right)\right]$ (when $\hbar \omega_{0}$ is the optical phonon energy, $E$ is the maximum energy an Auger electron can lose and still be able to surmount the heteiobarrier, and $L_{P}$ is the electron mean free path). Due to the uncertainty in $L_{P}$ and the availability of experimental data on carrier heating effect, the former is chosen. The results of $T_{e}: t 300 \mathrm{~K}$ from [12] have been used and the electron temperatures at various lattice temperatures $\left(T_{L}\right)$ are estimated from the expression

$$
T_{e}\left(T_{L}\right)=T_{e}(300) \frac{C_{\text {chcc }}\left(T_{L}\right)}{C_{\text {chcc }}(300)} .
$$

Thus, it is implicitly assumed that the hot carriers are generated by the Auger process.

Results of the calculation are presented in Figs. 2-4. Fig. 2 shows the calculated ratio of $J_{e} / J_{p}$ in an LED with $d=0.15$ $\mu \mathrm{m}, L=0.3 \mu \mathrm{m}$ at various temperatures under three different injection conditions. The triangles are experimental data from [2]. For $\Delta E_{c}=\frac{1}{3} \Delta E_{g}$, reasonable agreement can be obtained by taking $N_{A 1}=4.6 \times 10^{17} \mathrm{~cm}^{-3}$ and $N_{A 2}=1.4 \times 10^{17} \mathrm{~cm}^{-3}$.

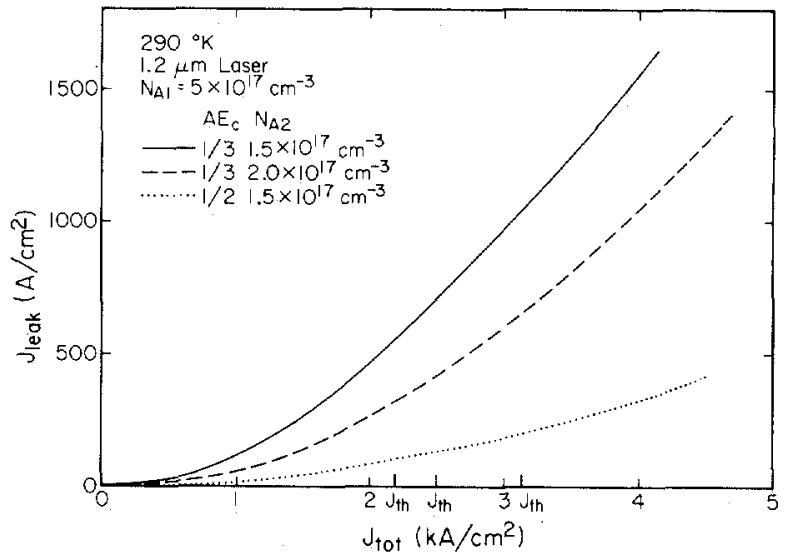

Fig. 3. Calculated electron leakage current versus total injected current for different P-InP doping levels and different discontinuities $\left(\Delta E_{c}\right)$ in the conduction band.

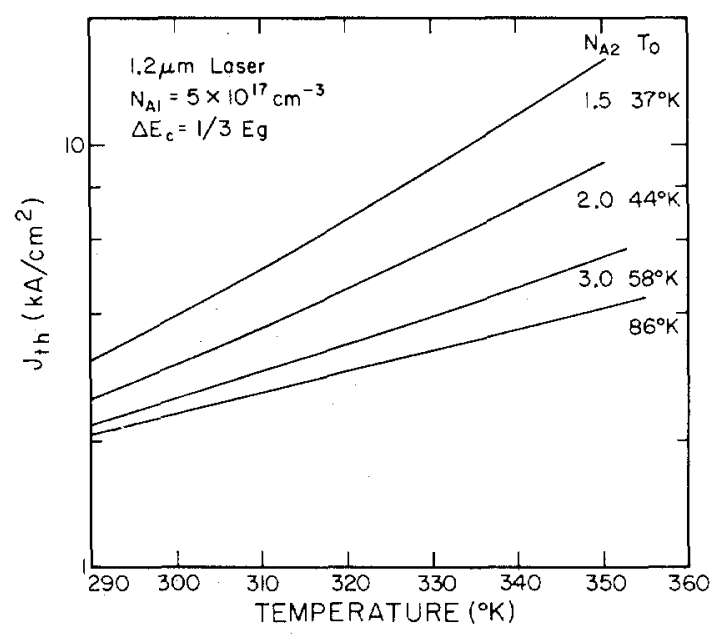

Fig. 4. Calculated $T_{o}$ for different P-InP doping levels. The $T_{o}=86 \mathrm{~K}$ is calculated without electron leakage.

The dashed line denotes the ratio of $J_{e} / J_{p}$ when carrier heating effects have been neglected. Note that the temperature dependence of $J_{e} / J_{p}$ remains the same when (12) is used. Fig. 3 shows the calculated effect of doping and discontinuity on the magnitude of leakage current in a $1.2 \mu \mathrm{m}$ laser with $d=0.2 \mu \mathrm{m}$ and $L=1.5 \mu \mathrm{m}$. It is apparent that in order to minimize leakage, the cladding layer could be heavily doped or a graded junction be grown to smear the "spike" at the interface. There is a slight discontinuity in slope at $J_{\mathrm{th}}$. However, for larger leakage currents, the change in slope is very small and insignificant. Fig. 4 depicts the effect of leakage current on the $T_{o}$ of the laser. The influence of the P-doping is again conspicuous. The general trend is in agreement with the experimental results of $\mathrm{Ng}$ et al. [6] and Mito et al. [22].

In lasers, as a comparison, for $\Delta E_{c}=\frac{1}{3} \Delta E_{g}, N_{A 1}=5 \times 10^{17}$ $\mathrm{cm}^{-3}$, and $N_{A 2}=2 \times 10^{17} \mathrm{~cm}^{-3}$; the ratios of $J_{e} / J_{\text {tot }}$ at an ambient temperature of $290 \mathrm{~K}$ for $1.2,1.3$, and $1.5 \mu \mathrm{m}$ devices are estimated to be 22,11 , and 4 percent, respectively, when carrier heating effects [12] are taken into account. The corresponding figures are 11,3 , and 0.4 percent when hot carriers are absent. Thus, it can be seen that carrier heating effects 
play an important role in device performance, especially for wavelengths beyond $1.3 \mu \mathrm{m}$.

In conclusion, a model calculation for the field and hot carrier enhanced electron leakage over the heterobarrier in InGaAsP/InP LED's and lasers has been presented. Results reveal the importance of the doping level in the P-InP confining layer in determining the magnitude of the leakage current.

\section{REFERENCES}

[1] S. Yamakoshi, T. Sanada, O. Wada, I. Umebu, and T. Sakurai, "Direct observation of electron leakage in InGaAsP/InP double heterostructure," Appl. Phys. Lett., vol. 40, pp. 144-146, 1982.

[2] - "Effect of carrier leakage on InGaAsP/InP double-heterostructure light emitters," in Proc. Int. Conf. Gallium Arsenide and Related Compounds, 1981 Oiso, Inst. Phys. Conf. Ser. 63, p. 485 .

[3] T. R. Chen, S. Margalit, U. Koren, K. L. Yu, L. C. Chiu, A. Hasson, and A. Yariv, "Direct measurement of the carrier leakage in InGaAsP/InP laser," Appl. Phys. Lett., vol. 42, pp. 1000-1002, 1983.

[4] C. M. Wu and E. S. Yang, "Physical mechanisms of carrier leakage in KH injection lasers," J. Appl. Phys., vol. 49, pp. 3114-3117, 1978.

[5] M. Yano, H. Imai, and M. Takusagawa, "Analysis of electrical, threshold, and temperature characteristics of InGaAsP/InP doubleheterojunction lasers," IEEE $J$. Quantum Electron., vol. QE-17, pp. 1954-1964, 1981.

[6] W. W. Ng and P. D. Dapkus, "Growth and characterization of $1.3 \mu \mathrm{m} \mathrm{CW} \mathrm{GaInAsP/InP} \mathrm{lasers} \mathrm{by} \mathrm{liquid} \mathrm{phase} \mathrm{epitaxy,"} \mathrm{IEEE} J$. Quantum Electron., vol. QE-17, pp. 193-198, 1981.

[7] T. P. Lee and A. G. Dentai, "Power and modulation bandwidth of GaAs-AlGaAs high radiance LED's for optical communication systems," IEEE J. Quantum Electron., vol. QE-14, pp. 150-159, 1978.

[8] P. J. Anthony and N. E. Schumaker, "Ambipolar transport in double heterostructure injection lasers," IEEE Electron Device Lett., vol. EDL-1, pp. 58-60, 1980.
[9] —-, "Temperature dependence of the lasing threshold current of double heterostructure injection lasers due to drift current loss," J. Appl. Phys., vol. 51, pp. 5038-5040, 1980.

[10] D. L. Scharfetter, "Minority carrier injection and charge storage in epitaxial Schottky barrier diodes," Solid-State Electron., vol. 8, pp. 299-311, 1965.

[11] J. Shah, R. F. Leheny, R. E. Nahory, and H. Temkin, "Hotcarrier effects in 1.3- $\mathrm{In}_{1-x} \mathrm{Ga}_{x} \mathrm{As}_{y} \mathrm{P}_{1-y}$ light emitting diodes," Appl. Phys. Lett., vol. 39, pp. 618-620, 1981.

[12] O. Wada, S. Yamakoshi, and T. Sakurai, "Band-gap enhanced carrier heating in InGaAsP/InP double heterostructure lightemitting diodes," Appl. Phys. Lett., vol. 41, pp. 981-983, 1982.

[13] J. R. Hayes, A. R. Adams, and P. D. Greene, "Mobility of holes in the quaternary alloy $\mathrm{In}_{1-x} \mathrm{Ga}_{x} \mathrm{As}_{y}, \mathrm{P}_{1-y}$," Electron. Lett., vol. 16 , pp. $282-284,1980$.

[14] H. C. Casey, Jr. and M. B. Panish, Heterostructure Lasers. New York: Academic, 1978, pt. A, ch. 4.

[15] S. M. Sze, Physics of Semiconductor Devices. New York: Wiley, 1981, ch. 5.

[16] L. L. Chang, "The conduction properties of Ge-GaAs ${ }_{1-x} P_{x} n-n$ heterojunctions," Solid-state Electron., vol. 8, pp. 721-728, 1965.

[17] L. C. Chiu, P. C. Chen, and A. Yariv, "Interband Auger recombination in InGaAsP," IEEE J. Quantum Electron., vol. QE-18, pp. $938-941,1982$

[18] S. R. Forrest, O. K. Kim, and R. G. Smith, "Optical response time of $\mathrm{In}_{0.53} \mathrm{Ga}_{0.47} \mathrm{As} / \mathrm{InP}$ avalanche photodiodes," Appl. Phys. Lett., vol. 41 , pp. $95-98,1982$.

[19] G. E. Stillman, L. W. Cook, G. E. Bulman, N. Tabatabaier, R. Chin, and P. D. Dapkus, "Long-wavelength $(1.3-$ to $1.6-\mu \mathrm{m}) \mathrm{de}-$ tectors for fiber-optical communications," IEEE Trans. Electron Devices, vol. ED-29, pp. 1355-1371, 1982.

[20] W. R. Frensley and H. Kroemer, "Theory of the energy-band lineup at an abrupt semiconductor heterojunction," Phys. Rev. B, vol. 16, pp. 2642-2652, 1977.

[21] L. C. Chiu and A. Yariv, "Comments on the nature of electron leakage in InGaAsP/InP double heterostructure," Japan. J. Appl. Phys., vol. 21, pp. L305-L306, 1982.

[22] I. Mito, M. Kitamura, K. Kobayashi, K. Kobayashi, and R. Lang, "High $T_{o}$ and high temperature CW operation in InGaAsP DCPBH LD," presented at 8th IEEE Int. Semicond. Laser Conf., Ottawa, Ont., Canada, 1982, Paper 28. 